\title{
Glycemic Control and Neonatal Outcomes in Women With Gestational Diabetes Mellitus Treated Using Glyburide, Metformin, or Insulin: A Pairwise and Network Meta-Analysis
}

\author{
Dan-Qing Yu \\ Second Affiliated Hospital of Zhejiang University \\ Guan-Xin Xu \\ Second Affiliated Hospital of Zhejiang University \\ Xin-Yuan Teng \\ Second Affiliated Hospital of Zhejiang University \\ Jing-Wei $X u$ \\ Second Affiliated Hospital of Zhejiang University \\ Liang-Fang Tang \\ Second Affiliated Hospital of Zhejiang University \\ Chun Feng \\ Second Affiliated Hospital of Zhejiang University \\ Jin-Peng Rao \\ Second Affiliated Hospital of Zhejiang University \\ Min Jin \\ Second Affiliated Hospital of Zhejiang University \\ Li-Quan Wang ( $\square$ wangliquan@zju.edu.cn ) \\ Second Affiliated Hospital of Zhejiang University
}

\section{Research Article}

Keywords: network meta-analysis, gestational diabetes mellitus, metformin, glyburide, insulin

Posted Date: January 22nd, 2021

DOI: https://doi.org/10.21203/rs.3.rs-146509/v1

License: () (1) This work is licensed under a Creative Commons Attribution 4.0 International License. Read Full License 


\section{Abstract}

Aims: We aimed to assess the comparative efficiency and safety of the use of glyburide, metformin, and insulin in gestational diabetes mellitus (GDM).

Methods: We searched for randomized controlled trials that compared glyburide, metformin, and insulin in GDM. Data regarding glycemic control and neonatal safety were collected and analyzed in pairwise and network meta-analyses.

Results: A total of 4,683 individuals from 24 trials were included. Compared with glyburide, metformin reduced 2-hour postprandial blood glucose (2HPG) to a greater extent (standard mean difference (SMD) $0.18 ; 95 \%$ credible interval (CI) $0-0.36$ ). There were significantly lower prevalences of neonatal hypoglycemia (risk difference (RD) $-0.07 ; 95 \% \mathrm{Cl}-0.11--0.03$ ), neonatal intensive care unit (NICU) admission (RD-0.03; 95\% Cl-0.06--0), and preeclampsia (RD-0.03; $95 \% \mathrm{Cl}-0.06--0)$ in the metformin group than in the insulin group. The metformin group had significantly lower birth weight (SMD-0.25; $95 \% \mathrm{Cl}-0.41--0.10)$ and maternal weight gain (SMD-0.61; $95 \% \mathrm{Cl}-0.86--0.35)$ compared with the insulin group. Network meta-analysis suggested that metformin had the highest probability of successfully controlling glycemia and preventing neonatal complications.

Conclusions: The present meta-analysis suggests that metformin may be as effective as insulin for glycemic control and is the most promising drug for the prevention of neonatal and maternal complications.

\section{Introduction}

Gestational diabetes mellitus (GDM) is defined in the World Health Organization guidelines as glucose intolerance or hyperglycemia that occurs or is first recognized during pregnancy. Owing to changes in lifestyle and increasing maternal age, the prevalence of GDM has increased markedly during the past two decades, with prevalences of between $1 \%$ and $26 \%$ being recorded in different studies [1-6].

GDM can lead to maternal and neonatal outcomes in both the short and long term. The maternal complications include preeclampsia (PE), cesarean section, and polyhydramnios in the short term, and the progression of diabetes mellitus after pregnancy in the long term [1]. The fetal and neonatal complications include congenital malformation, neonatal death, stillbirth, macrosomia, obstetric trauma, shoulder dystocia, and neonatal hypoglycemia [7, 8].

Appropriate glycemic control is the principal means of preventing hyperinsulinemia and macrosomia [9]. As the conventional treatment for GDM, insulin is effective and safe. However, it is inconvenient and expensive to use. During the last 20 years, oral hypoglycemic agents (OHAs) have been introduced for GDM, of which the most frequently used drugs are glyburide and metformin. Glyburide is a second-generation sulfonylurea that binds to receptors on beta-cells and increases the secretion of insulin. Metformin is a biguanide that increases insulin-stimulated glucose uptake in muscle cells, and suppresses gluconeogenesis and fatty acid oxidation in hepatocytes. Because they are cheap and convenient, OHAs have been used by increasing numbers of patients and health care providers. In the USA, glyburide has been widely used for the treatment of GDM, whereas in Europe it has not been routinely used to treat GDM. Metformin is labeled as a category B drug, meaning that there is no strong evidence that it causes primary fetal malformation. Therefore, it has been commonly used for the treatment of polycystic ovary syndrome $[10,11]$. Although there is an increasing amount of evidence that supports the use of glyburide or metformin for GDM, the American Diabetes Association (ADA) and American College of Obstetricians and Gynecologists (ACOG) still recommend insulin as the first-line treatment, due to the lack of evidence regarding the long-term safety of the alternatives [12, 13].

Both glyburide and metformin can pass across the placenta [14-16], and therefore there is potential for adverse effects on the fetus. Recent studies and metaanalyses have shown higher prevalences of neonatal complications, mainly macrosomia and neonatal hypoglycemia, in users of glyburide [17-24], and higher prevalences of preterm birth and preeclampsia in users of metformin [25-28], compared with users of insulin.

In 2019, two pairwise meta-analyses were conducted of glycemic control and neonatal outcomes. Both of these showed significant heterogeneity in glycemic control $[29,30]$. However, because these studies used blood glucose concentration in the third trimester as the index of glycemic control, the heterogeneity may at least, in part, have been due to the differing baseline glucose concentrations in different populations and among individuals with different lifestyles. In the present study, we calculated the changes in FBG, 2HPG, and HbA1c that occurred from baseline during treatment. This approach was intended to limit the heterogeneity, such that the indices would more accurately reflect the effects of treatment.

The aim of the present study was to identify all the RCTs that have compared the use of glyburide, metformin, and insulin to treat GDM and conduct a network meta-analysis to compare the effects of each on glycemic control and neonatal outcomes, as well as their safety. In this way, we aimed to provide evidence for the rational treatment of GDM in clinical practice.

\section{Research Design And Methods}

We conducted this study according to the Cochrane Handbook for the Systematic Review of Interventions and the Guidelines of the Preferred Reporting Items for Systematic Review and Meta-Analysis Protocols (PRISMA, version 6). This meta-analysis was registered with PROSPERO (number CRD42020178575).

\subsection{Inclusion and exclusion criteria}

We considered RCTs of GDM that compared any of the following treatments: glyburide, metformin, and insulin. Trials of dietary control and exercise may have included participants with differing baseline blood glucose concentrations, and were therefore not considered. Literature searches were conducted in PubMed, Web of Science, EBSCO, and Scopus. Two assessors screened the publications independently on April 7, 2020. The search terms used were 'gestational diabetes' or 'GDM'; 'insulin', or 'oral hypoglycemic agents', or 'oral antidiabetic drugs', or 'glibenclamide', or 'glyburide', or 'metformin'; and 'randomized 
controlled trial'. The searches were limited to English language publications. We also checked the reference lists of the included studies for further relevant trials.

Trials in which patients were enrolled who developed diabetes before gestation were excluded. Trials that only included patients with mild GDM were also excluded; this is because we were concerned about the effects of pharmacotherapy in patients with poor glycemic control after medical nutritional therapy. Furthermore, studies were excluded that contained only per-protocol (PP) analyses or in which the baseline characteristics, except for glycemic control parameters, were not well-matched.

\subsection{Assessment of the risk of bias in the included studies}

Two authors independently assessed the risk of bias using the Cochrane Handbook for Systematic Reviews of Interventions. Any disagreement between the authors was resolved by discussion.

\subsection{Outcomes}

Estimates of the mean changes and standard deviations from baseline of FBG, 2HPG, and HbA1c were reported as effects of the treatments on glycemic control. They were calculated using the means and standard deviations of the baseline and post-treatment values, using the recommended approach [31, 32].

The neonatal outcomes were anomalies, neonatal hyperbilirubinemia, neonatal hypoglycemia, neonatal intensive care unit (NICU) admission, respiratory distress syndrome (RDS), obstetric trauma, preterm birth, perinatal death, birth weight, macrosomia, large-for-gestational age (LGA) fetus, and small-forgestational age (SGA) fetus. The maternal outcomes were preeclampsia and maternal weight gain from the time of enrollment.

\subsection{Data synthesis and statistical analysis}

In trials that reported findings in participants who were treated with metformin alone or metformin and supplementary insulin, the mean and SD of the metformin group was calculated from the data from the divided subgroups, as previously reported [33]. Glyburide- and glibenclamide-treated participants were considered together in the present analysis.

Pairwise meta-analysis was conducted using Review Manager (version 5.3). We conducted pairwise meta-analysis with a random-effect model if significant heterogeneity was identified; otherwise, a fixed-effect model was used. The Studies were determined to be heterogeneous if $\mathrm{I}^{2}>50 \%$ and $P<0.1$. The standard mean difference (SMD) was calculated using the mean and SD for continuous variables and the risk difference (RD) was calculated for dichotomous variables. Both are quoted with $95 \%$ credible intervals (Cls).

Glycemic control and neonatal and maternal safety outcomes were synthesized using network meta-analysis (NMA) in a Bayesian multilevel framework. NMA was performed using Stata (version 15). We present the results as a ranking probability, using their surface under the cumulative ranking curve (SUCRA), for different efficiency and safety outcomes. For each network analysis, we assessed the consistency of direct and indirect comparisons using node-splitting analysis, with $p \leq 0.05$ indicating significant inconsistency. The results indicated that the direct and indirect comparisons were consistent for all the NMAs, and the results of the NMAs are shown using consistency models.

\section{Results}

\subsection{Characterization of the included trials}

A total of 1,708 records were identified through the database searches, of which 1,196 records remained after the removal of duplicates. Thirty-four studies met the inclusion criteria, of which three included duplication with regard to follow-up data [34-36]. Therefore, the later publications were not included in the final analysis. The study and patient characteristics of the 31 remaining studies are summarized in Table 1 . The screening protocols and diagnostic criteria for GDM, and the details of the funding and conflict of interest are summarized in Supplementary table $1[25,26,28,37-63]$. Insulin was most frequently prescribed as a combination of rapid-acting and intermediate insulin (Supplementary table 1). The most frequently used targets for glucose control were FBG $<90-100 \mathrm{mg} / \mathrm{dl}$ and $2 \mathrm{HPG}<120-126 \mathrm{mg} / \mathrm{dl}$ (Supplementary table 1). Of these 31 studies, four reported data according to the per-protocol (PP) principle[46, $52,57,62]$ and one did not report the principle of the data analysis [38]. Three studies reported results according to both the intention-to-treat (ITT) and PP principles $[40,41,55]$ because no patient drop out from the group after randomization, and one study reported both ITT and PP data [61]. Therefore, the four studies that were conducted according to an unknown principle or only used the PP principle were not included in quantitative analysis. As shown in Table 1 , one study showed a significant difference in maternal age between the groups at baseline [55] and another showed a significant difference in gestational age at enrollment [42], which were not included in the meta-analysis because these two parameters have direct effects on the efficacy of treatment. A PRISMA flow chart that summarizes the search results and the trials included in the analysis is provided in Supplementary Fig. 1. The risks of bias in the 24 studies included in the meta-analysis are summarized in Supplementary Figs. 2 and 3. 
Table 1

Study and patient characteristics of included trials.

\begin{tabular}{|c|c|c|c|c|c|c|c|c|c|c|c|c|c|}
\hline \multirow[t]{2}{*}{$\begin{array}{l}\text { Study (author } \\
\text { year) }\end{array}$} & \multirow[t]{2}{*}{ Country } & \multirow[t]{2}{*}{$\begin{array}{l}\text { Principle } \\
\text { of data } \\
\text { analysis }\end{array}$} & \multicolumn{2}{|l|}{ Drug } & \multicolumn{2}{|l|}{ Sizes } & \multicolumn{2}{|l|}{ BMI } & \multicolumn{2}{|l|}{ Age } & \multicolumn{2}{|c|}{$\begin{array}{l}\text { Gestational } \\
\text { age at } \\
\text { enrollment }\end{array}$} & \multirow[t]{2}{*}{$\begin{array}{l}\text { Includ } \\
\text { in met } \\
\text { analy! }\end{array}$} \\
\hline & & & Group 1 & Group 2 & Group 1 & $\begin{array}{l}\text { Group } \\
2\end{array}$ & Group & $\begin{array}{l}\text { Group } \\
2\end{array}$ & $\begin{array}{l}\text { Group } \\
1\end{array}$ & $\begin{array}{l}\text { Group } \\
2\end{array}$ & $\begin{array}{l}\text { Group } \\
1\end{array}$ & Group & \\
\hline Rowan 2003 & NA & NA & Metformin & Insulin & 16 & 14 & $\begin{array}{l}39.5 \\
\pm 6.94\end{array}$ & $\begin{array}{l}37.9 \\
\pm 6.87\end{array}$ & $\begin{array}{l}33.7 \\
\pm 4.44\end{array}$ & $\begin{array}{l}34.1 \\
\pm 3.7\end{array}$ & $\begin{array}{l}29.8 \\
\pm 4.49\end{array}$ & $\begin{array}{l}30.4 \\
\pm 4.67\end{array}$ & No \\
\hline Moore 2007 & USA & ITT/PP & Metformin & Insulin & 32 & 31 & $\begin{array}{l}39.7 \\
\pm 9\end{array}$ & $\begin{array}{l}35.3 \\
\pm 6.7\end{array}$ & $\begin{array}{l}27.1 \\
\pm 4.7\end{array}$ & $\begin{array}{l}27.7 \\
\pm 6.7\end{array}$ & $\begin{array}{l}27.8 \\
\pm 6.5\end{array}$ & $\begin{array}{l}28.9 \\
\pm 5\end{array}$ & Yes \\
\hline Rowan 2008 & $\begin{array}{l}\text { New } \\
\text { Zealand, } \\
\text { Australia }\end{array}$ & ITT & Metformin & Insulin & 363 & 370 & $\begin{array}{l}35.1 \\
\pm 8.3\end{array}$ & $\begin{array}{l}34.6 \\
\pm 7.2\end{array}$ & $\begin{array}{l}33.5 \\
\pm 5.4\end{array}$ & $\begin{array}{l}33 \pm \\
5.1\end{array}$ & $\begin{array}{l}30.2 \\
\pm 3.3\end{array}$ & $\begin{array}{l}30.1 \\
\pm 3.2\end{array}$ & Yes \\
\hline ljas 2010 & Finland & ITT & Metformin & Insulin & 47 & 50 & $\begin{array}{l}31.5 \\
\pm 6.5\end{array}$ & $\begin{array}{l}30.8 \\
\pm 5.4\end{array}$ & $\begin{array}{l}32.3 \\
\pm 5.6\end{array}$ & $\begin{array}{l}31.7 \\
\pm 6.1\end{array}$ & $\begin{array}{l}30 \pm \\
4.9\end{array}$ & $30 \pm 4$ & Yes \\
\hline $\begin{array}{l}\text { Niromanesh } \\
2012\end{array}$ & Iran & ITT & Metformin & Insulin & 80 & 80 & $\begin{array}{l}28.1 \\
\pm 4\end{array}$ & $\begin{array}{l}27.1 \\
\pm 2.1\end{array}$ & $\begin{array}{l}30.7 \\
\pm 5.5\end{array}$ & $\begin{array}{l}31.8 \\
\pm 5.1\end{array}$ & $\begin{array}{l}28.7 \\
\pm 3.7\end{array}$ & $\begin{array}{l}28.6 \\
\pm 3.6\end{array}$ & Yes \\
\hline $\begin{array}{l}\text { Mesdaghinia } \\
2012\end{array}$ & Iran & PP & Metformin & Insulin & 100 & 100 & $\begin{array}{l}27.6 \\
\pm \text { NA }\end{array}$ & $\begin{array}{l}28.46 \\
\pm \mathrm{NA}\end{array}$ & $\begin{array}{l}29.6 \\
\pm 5.3\end{array}$ & $\begin{array}{l}30.2 \\
\pm 5.9\end{array}$ & $\begin{array}{l}27.9 \\
\pm 3.22\end{array}$ & $\begin{array}{l}28.9 \\
\pm 3.8\end{array}$ & No \\
\hline Hassan 2012 & Pakistan & ITT & Metformin & Insulin & 75 & 75 & $\begin{array}{l}29.17 \\
\pm 1.94\end{array}$ & $\begin{array}{l}28.74 \\
\pm 2.69\end{array}$ & $\begin{array}{l}30.29 \\
\pm 3.06\end{array}$ & $\begin{array}{l}30.88 \\
\pm 3.6\end{array}$ & $\begin{array}{l}29.53 \\
\pm 1.33\end{array}$ & $\begin{array}{l}29.2 \\
\pm 1.48\end{array}$ & Yes \\
\hline $\begin{array}{l}\text { Spaulonei } \\
2013\end{array}$ & Brazil & ITT & Metformin & Insulin & 46 & 46 & $\begin{array}{l}31.96 \\
\pm 4.75\end{array}$ & $\begin{array}{l}31.39 \\
\pm 5.71\end{array}$ & $\begin{array}{l}31.93 \\
\pm 6.02\end{array}$ & $\begin{array}{l}32.76 \\
\pm 4.66\end{array}$ & $\begin{array}{l}32.18 \\
\pm 3.7\end{array}$ & $\begin{array}{l}32.05 \\
\pm 3.5\end{array}$ & Yes \\
\hline Tertti 2013 & Finland & ITT & Metformin & Insulin & 110 & 107 & $\begin{array}{l}29.4 \\
\pm 5.9\end{array}$ & $\begin{array}{l}28.9 \\
\pm 4.7\end{array}$ & $\begin{array}{l}31.9 \\
\pm 5\end{array}$ & $\begin{array}{l}32.1 \\
\pm 5.4\end{array}$ & $\begin{array}{l}30.3 \\
\pm 2\end{array}$ & $\begin{array}{l}30.4 \\
\pm 1.8\end{array}$ & Yes \\
\hline $\begin{array}{l}\text { Ruholamin } \\
2014\end{array}$ & Iran & PP & Metformin & Insulin & 50 & 50 & $\begin{array}{l}26.4 \\
\pm 2.8\end{array}$ & $\begin{array}{l}25.1 \\
\pm 3.4\end{array}$ & $\begin{array}{l}24.6 \\
\pm 6.3\end{array}$ & $\begin{array}{l}23.4 \\
\pm 2.5\end{array}$ & $\begin{array}{l}27.6 \\
\pm 3.3\end{array}$ & $\begin{array}{l}26.7 \\
\pm 3.5\end{array}$ & No \\
\hline Ainuddin 2014 & Pakistan & ITT & Metformin & Insulin & 75 & 75 & NA & NA & $\begin{array}{l}30.34 \\
\pm 3.07\end{array}$ & $31 \pm 4$ & NA & NA & Yes \\
\hline Ashoush 2016 & Egypt & ITT & Metformin & Insulin & 47 & 48 & $\begin{array}{l}31.1 \\
\pm 1.3\end{array}$ & $\begin{array}{l}31.4 \\
\pm 1.5\end{array}$ & $\begin{array}{l}31.6 \\
\pm 2.8\end{array}$ & $\begin{array}{l}32.1 \\
\pm 3.2\end{array}$ & $\begin{array}{l}29.8 \\
\pm 1.4\end{array}$ & $\begin{array}{l}29.7 \\
\pm 1.9\end{array}$ & Yes \\
\hline Saleh 2016 & Egypt & ITT & Metformin & Insulin & 67 & 70 & $\begin{array}{l}30.52 \\
\pm 3.17\end{array}$ & $\begin{array}{l}31.58 \\
\pm \\
30.12\end{array}$ & $\begin{array}{l}31 \pm \\
3.42\end{array}$ & $\begin{array}{l}29.8 \\
\pm 2.18\end{array}$ & NA & NA & Yes \\
\hline Arshad 2017 & Pakistan & ITT & Metformin & Insulin & 25 & 25 & NA & NA & $\begin{array}{l}29.76 \\
\pm 3.41\end{array}$ & $\begin{array}{l}31.6 \\
\pm 4.27\end{array}$ & NA & NA & Yes \\
\hline Gamal 2018 & Egypt & ITT & Metformin & Insulin & 58 & 58 & $\begin{array}{l}29.6 \\
\pm 1.3\end{array}$ & $\begin{array}{l}29.4 \\
\pm 1.4\end{array}$ & $\begin{array}{l}30.4 \\
\pm 2.8\end{array}$ & $\begin{array}{l}30.6 \\
\pm 2.5\end{array}$ & $\begin{array}{l}28.9 \\
\pm 1.1\end{array}$ & $\begin{array}{l}29 \pm \\
1.1\end{array}$ & Yes \\
\hline Ghomian 2019 & Iran & PP & Metformin & Insulin & 143 & 143 & NA & NA & NA & NA & NA & NA & No \\
\hline Wasim 2019 & Pakistan & ITT & Metformin & Insulin & 137 & 141 & NA & NA & $\begin{array}{l}29.5 \\
\pm 4.8\end{array}$ & $\begin{array}{l}29.7 \\
\pm 4.8\end{array}$ & $\begin{array}{l}28.9 \\
\pm 2.9\end{array}$ & $\begin{array}{l}28.6 \\
\pm 3.1\end{array}$ & Yes \\
\hline Langer 2000 & USA & ITT & Glyburide & Insulin & 201 & 203 & NA & NA & $29 \pm 7$ & $30 \pm 6$ & $24 \pm 7$ & $25 \pm 7$ & Yes \\
\hline Bertini 2005 & Brazil & ITT & Glyburide & Insulin & 24 & 27 & $\begin{array}{l}27.5 \\
\pm 5.8\end{array}$ & $\begin{array}{l}27 \pm \\
7.2\end{array}$ & $\begin{array}{l}31.2 \\
\pm 4.5\end{array}$ & $\begin{array}{l}28.7 \\
\pm 6\end{array}$ & NA & NA & Yes \\
\hline $\begin{array}{l}\text { Anjalakshi } \\
2007\end{array}$ & India & ITT/PP & Glyburide & Insulin & 10 & 13 & $\begin{array}{l}22.82 \\
\pm 3.5\end{array}$ & $\begin{array}{l}25.32 \\
\pm 5.14\end{array}$ & $\begin{array}{l}24.9 \\
\pm 3.76\end{array}$ & $\begin{array}{l}27.46 \\
\pm 5.83\end{array}$ & $\begin{array}{l}22.5 \\
\pm 4.72\end{array}$ & $\begin{array}{l}22.62 \\
\pm 5.62\end{array}$ & Yes \\
\hline $\begin{array}{l}\text { Ogunyemi } \\
2007\end{array}$ & USA & ITT & Glyburide & Insulin & 48 & 49 & $\begin{array}{l}32 \pm \\
7.6\end{array}$ & $\begin{array}{l}30.8 \\
\pm 6.9\end{array}$ & NA & NA & $\begin{array}{l}28.1 \\
\pm 7.6 *\end{array}$ & $\begin{array}{l}24.6 \\
\pm 8^{*}\end{array}$ & No \\
\hline Lain 2009 & USA & ITT & Glyburide & Insulin & 41 & 41 & $\begin{array}{l}33.4 \\
\pm 12.9\end{array}$ & $\begin{array}{l}30.9 \\
\pm 5.7\end{array}$ & $\begin{array}{l}32.2 \\
\pm 5\end{array}$ & $\begin{array}{l}31.2 \\
\pm 5.9\end{array}$ & $\begin{array}{l}30.8 \\
\pm 2.5\end{array}$ & $\begin{array}{l}30.6 \\
\pm 2.2\end{array}$ & Yes \\
\hline $\begin{array}{l}\text { Mukhopadhyay } \\
2012\end{array}$ & India & ITT & Glyburide & Insulin & 30 & 30 & $\begin{array}{l}23.7 \\
\pm 2.7\end{array}$ & $\begin{array}{l}23 \pm \\
2.9\end{array}$ & $\begin{array}{l}26.3 \\
\pm 4.6\end{array}$ & $\begin{array}{l}26 \pm \\
4.3\end{array}$ & $\begin{array}{l}28.3 \\
\pm 2.2\end{array}$ & $\begin{array}{l}27.4 \\
\pm 2.7\end{array}$ & Yes \\
\hline Tempe 2013 & India & ITT & Glyburide & Insulin & 32 & 32 & NA & NA & NA & NA & NA & NA & Yes \\
\hline $\begin{array}{l}\text { Mirzamoradi } \\
2015\end{array}$ & Iran & ITT/PP & Glyburide & Insulin & 37 & 59 & NA & NA & $\begin{array}{l}29.5 \\
\pm \\
4.06 *\end{array}$ & $\begin{array}{l}31.18 \\
\pm \\
5.01 *\end{array}$ & $\begin{array}{l}29.89 \\
\pm 4.12\end{array}$ & $\begin{array}{l}30.27 \\
\pm 3.97\end{array}$ & No \\
\hline Bhrashi 2016 & Iran & PP & Glyburide & Insulin & 120 & 129 & $\begin{array}{l}21.94 \\
\pm 2.8\end{array}$ & $\begin{array}{l}22.59 \\
\pm \\
3.094\end{array}$ & $\begin{array}{l}30.69 \\
\pm \\
7.194\end{array}$ & $\begin{array}{l}29.98 \\
\pm \\
7.033\end{array}$ & $\begin{array}{l}24.89 \\
\pm 3.9\end{array}$ & $\begin{array}{l}24.48 \\
\pm 4.51\end{array}$ & No \\
\hline
\end{tabular}




\begin{tabular}{|c|c|c|c|c|c|c|c|c|c|c|c|c|c|}
\hline \multirow[t]{2}{*}{$\begin{array}{l}\text { Study (author } \\
\text { year) }\end{array}$} & \multirow[t]{2}{*}{ Country } & \multirow[t]{2}{*}{$\begin{array}{l}\text { Principle } \\
\text { of data } \\
\text { analysis }\end{array}$} & \multicolumn{2}{|l|}{ Drug } & \multicolumn{2}{|l|}{ Sizes } & \multicolumn{2}{|l|}{ BMI } & \multicolumn{2}{|l|}{ Age } & \multicolumn{2}{|c|}{$\begin{array}{l}\text { Gestational } \\
\text { age at } \\
\text { enrollment }\end{array}$} & \multirow[t]{2}{*}{$\begin{array}{l}\text { Includ } \\
\text { in met } \\
\text { analys }\end{array}$} \\
\hline & & & Group 1 & Group 2 & Group 1 & $\begin{array}{l}\text { Group } \\
2\end{array}$ & $\begin{array}{l}\text { Group } \\
1\end{array}$ & $\begin{array}{l}\text { Group } \\
2\end{array}$ & $\begin{array}{l}\text { Group } \\
1\end{array}$ & $\begin{array}{l}\text { Group } \\
2\end{array}$ & $\begin{array}{l}\text { Group } \\
1\end{array}$ & $\begin{array}{l}\text { Group } \\
2\end{array}$ & \\
\hline Senat 2018 & France & PP/ITT & Glyburide & Insulin & 448(ITT) & 442 & $\begin{array}{l}30.7 \\
\pm 5.1\end{array}$ & $\begin{array}{l}31.1 \\
\pm 5.4\end{array}$ & $\begin{array}{l}32.5 \\
\pm 5.1\end{array}$ & $\begin{array}{l}32.6 \\
\pm 5.3\end{array}$ & NA & NA & Yes \\
\hline Moore 2009 & USA & ITT & Glyburide & Metformin & 74 & 75 & $\begin{array}{l}32.7 \\
\pm 7\end{array}$ & $\begin{array}{l}32.8 \\
\pm 5.8\end{array}$ & $\begin{array}{l}29.6 \\
\pm 7.8\end{array}$ & $\begin{array}{l}31 \pm \\
7.1\end{array}$ & $\begin{array}{l}29.1 \\
\pm 5\end{array}$ & $\begin{array}{l}27.3 \\
\pm 6.8\end{array}$ & Yes \\
\hline Silva 2012 & Brazil & ITT & Glyburide & Metformin & 96 & 104 & $\begin{array}{l}28.61 \\
\pm 5.88\end{array}$ & $\begin{array}{l}28.68 \\
\pm 5.37\end{array}$ & $\begin{array}{l}31.29 \\
\pm 5.36\end{array}$ & $\begin{array}{l}32.63 \\
\pm 5.61\end{array}$ & $\begin{array}{l}25.44 \\
\pm 7.13\end{array}$ & $\begin{array}{l}26.96 \\
\pm 6.44\end{array}$ & Yes \\
\hline George 2015 & India & ITT & Glyburide & Metformin & 53 & 51 & $\begin{array}{l}28.8 \\
\pm 4\end{array}$ & $\begin{array}{l}28.7 \\
\pm 4.4\end{array}$ & $\begin{array}{l}33.6 \\
\pm 4.6\end{array}$ & $\begin{array}{l}33.4 \\
\pm 4.4\end{array}$ & $\begin{array}{l}29.7 \\
\pm 3.7\end{array}$ & $\begin{array}{l}29.3 \\
\pm 3.3\end{array}$ & Yes \\
\hline Nachum 2017 & Israel & ITT & Glyburide & Metformin & 53 & 51 & $\begin{array}{l}28.6 \\
\pm 4.7\end{array}$ & $\begin{array}{l}28.6 \\
\pm 5.5\end{array}$ & $\begin{array}{l}32.8 \\
\pm 5\end{array}$ & $\begin{array}{l}33.6 \\
\pm 5.3\end{array}$ & $\begin{array}{l}29.4 \\
\pm 4\end{array}$ & $\begin{array}{l}29.6 \\
\pm 4.1\end{array}$ & Yes \\
\hline
\end{tabular}

\subsection{Glycemic control}

Of the 24 studies that were quantitatively analyzed, 20 reported both baseline and final glucose levels. The changes from baseline were calculated using the reported baseline and post-treatment blood glucose concentrations for ITT studies (Table 2). One study showed significantly higher baseline $2 \mathrm{HPG}$ (231.5 \pm $23.2 \mathrm{mg} / \mathrm{dl} v \mathrm{~s} .216 \pm 17 \mathrm{mg} / \mathrm{dl})$ and HbA1c $(5.36 \pm 0.478 \%$ vs. $5.2 \pm 0.58 \%)$ levels in the metformin group than in the insulin group [53], whereas another showed significantly lower baseline FBG in the metformin group than in the insulin group (104 $\pm 13.12 \mathrm{mg} / \mathrm{dl} v \mathrm{~s}$. $117.9 \pm 29.06 \mathrm{mg} / \mathrm{dl})$ [59]. Furthermore, Ogunyemi et al. reported significantly lower baseline FBG $(103.7 \pm 20.1 \mathrm{mg} / \mathrm{dl} v s .115 .8 \pm 19.6 \mathrm{mg} / \mathrm{dl})$ and HbA1c $(5.8 \pm 0.7 \%$ vs. $7.5 \pm 1.7 \%)$ levels in the glyburide group compared with the insulin group [42], and Mizamoradi et al. showed significantly lower 2HPG in the glyburide group than in the insulin group $(184.1 \pm 20.46 \mathrm{mg} / \mathrm{dl} v s .194 .3 \pm 18.47 \mathrm{mg} / \mathrm{dl})$ [55]. However, all of these four studies were included in the meta-analysis because we calculated the changes from baseline for FBG, $2 \mathrm{HPG}$, and $\mathrm{HbA} 1 \mathrm{c}$.

\subsection{FBG}

The results of the pairwise meta-analysis of glycemic control are shown in Supplementary Figs. 4-6. The reduction in FBG from baseline associated with glyburide treatment was significantly smaller than that associated with insulin treatment (SMD $0.18 ; 95 \% \mathrm{Cl} 0.01-0.34$ ). However, the reduction in FBG from baseline did not significantly differ between the metformin and insulin groups (SMD $0.06 ; 95 \% \mathrm{Cl}-0.03-0.16$ ) or the glyburide and metformin groups (SMD $0.05 ; 95 \% \mathrm{Cl}-0.23-0.13$ ). The NMA revealed no significances between metformin and insulin, glyburide and insulin, or glyburide and metformin (Table 3 ). Table 4 shows the treatment rankings, in which " 1 " is the least effective and " 3 " is the most effective. Metformin had the highest probability of being the least effective treatment for the reduction of FBG (64\%), while insulin had the highest probability of being the most effective treatment (76\%).

\subsection{HPG}

Pairwise meta-analysis of the change in 2HPG from baseline showed heterogeneity in the trials that compared metformin with insulin $\left(\mathrm{I}^{2}, 80 \%\right)$. The $2 \mathrm{HPG}$ reduction in the metformin group was larger than in the glyburide group ( $p=0.05$; SMD $0.18 ; 95 \% \mathrm{Cl} 0-0.36)$ (Supplementary Fig. 5). The NWA revealed no significant differences between the changes in 2HPG associated with each treatment (Table 3 ). With regard to the ranking probability, glyburide had the highest probability of being the least effective treatment (72\%), while metformin had the highest probability of being the most effective treatment (86\%) (Table 4). 
Table 2

Baseline and effect of treatment on FBG, $2 \mathrm{HPG}$ and $\mathrm{HbA} 1 \mathrm{c}$. * $P$ value $<0.05$, reported by the original study.

\begin{tabular}{|c|c|c|c|c|c|c|c|c|}
\hline \multirow{2}{*}{$\begin{array}{l}\text { Study } \\
\text { (author year) }\end{array}$} & \multirow[t]{2}{*}{ Drug } & \multicolumn{3}{|l|}{ FBG } & \multicolumn{3}{|l|}{$2 \mathrm{HPG}$} & \multirow{2}{*}{$\begin{array}{l}\text { HbA1c } \\
\text { Baseline } \\
\text { (SD) }\end{array}$} \\
\hline & & Baseline (SD) & $\begin{array}{l}\text { After } \\
\text { treatment } \\
\text { (SD) }\end{array}$ & $\begin{array}{l}\text { Change from } \\
\text { baseline (SD) }\end{array}$ & Baseline (SD) & $\begin{array}{l}\text { After } \\
\text { treatment } \\
\text { (SD) }\end{array}$ & $\begin{array}{l}\text { Change from } \\
\text { baseline (SD) }\end{array}$ & \\
\hline \multirow[t]{2}{*}{ Rowan 2008} & Metformin & $102.6(21.6)$ & $93.6(10.8)$ & $-9(18.7)$ & 174.6(37.8) & 111.6(10.8) & $-63(33.72)$ & $5.7(0.6)$ \\
\hline & Insulin & 102.6(19.8) & $91.8(12.6)$ & $-10.8(17.36)$ & 169.2(37.8) & $115.2(16.2)$ & $-54(32.85)$ & $5.8(0.7)$ \\
\hline \multirow{2}{*}{$\begin{array}{l}\text { Niromanesh } \\
2012\end{array}$} & Metformin & 104.7(8.6) & $88.3(7.7)$ & $-16.4(8.19)$ & $164.6(28.6)$ & 111.3(9.1) & $-53.3(25.31)$ & $5.7(0.6)$ \\
\hline & Insulin & 107.1(9.2) & $88.7(6.3)$ & $-18.4(8.15)$ & $172.5(30)$ & 111.1(9) & $-61.4(26.66)$ & $5.6(0.7)$ \\
\hline \multirow[t]{2}{*}{ Hassan 2012} & Metformin & 100.89 & NA & NA & 231.56 & NA & NA & $5.4(0.47)$ \\
\hline & Insulin & 102.11 & NA & NA & 236.41 & NA & NA & $5.19(0.59)$ \\
\hline \multirow[t]{2}{*}{$\begin{array}{l}\text { Spaulonei } \\
2013\end{array}$} & Metformin & 102.15(21.96) & $90.09(16.29)$ & $\begin{array}{l}-12.06 \\
(19.75)\end{array}$ & $124.17(24.4)$ & 108.44(13.39) & $-15.73(21.16)$ & $5.9(0.75)$ \\
\hline & Insulin & $\begin{array}{l}100.87 \\
(15.05)\end{array}$ & $88.35(7.45)$ & $\begin{array}{l}-12.52 \\
(13.03)\end{array}$ & $125.39(22.12)$ & 112.32(13.69) & $-13.07(19.34)$ & $5.93(0.8)$ \\
\hline \multirow[t]{2}{*}{ Tertti 2013} & Metformin & $99(9)$ & NA & NA & $149.4(32.4)$ & NA & NA & $5.48(0.34)$ \\
\hline & Insulin & $100.8(7.2)$ & NA & NA & $142.2(30.6)$ & NA & NA & $5.51(0.34)$ \\
\hline \multirow[t]{2}{*}{ Ainuddin 2014} & Metformin & $141(19)$ & $95.9(5.9)$ & $-45.1(16.84)$ & $231.547(23.2) *$ & 129.81(7.92) & $-101.737(20.426)$ & $5.36(0.478)$ * \\
\hline & Insulin & $172(215)$ & $97.4(2.5)$ & $\begin{array}{l}-74.6 \\
(213.761)\end{array}$ & $216(17) *$ & $128.1(6.4)$ & $-87.9(14.87)$ & $5.2(0.58)^{*}$ \\
\hline \multirow[t]{2}{*}{ Ashoush 2016} & Metformin & $105.7(4.7)$ & 78(3.1) & $-27.7(4.14)$ & $175.7(10)$ & 109.9(3.7) & $-65.8(8.757)$ & $5.7(0.5)$ \\
\hline & Insulin & $106.4(4.4)$ & $79.9(3.7)$ & $-26.5(4.1)$ & 177.6(8.8) & $111.3(4.2)$ & $-66.3(7.624)$ & $5.8(0.6)$ \\
\hline \multirow[t]{2}{*}{ Saleh 2016} & Metformin & $136.09(39.85)$ & $93.23(13.7)$ & $-42.86(35.07)$ & 198.32(214.67) & $116.52(3.53)$ & $-81.8(212.927)$ & NA \\
\hline & Insulin & 137.56(41.1) & $94.33(11.11)$ & $-43.23(36.82)$ & $196.52(15.45)$ & $117.12(3.45)$ & $-79.4(14.05)$ & NA \\
\hline \multirow[t]{2}{*}{ Arshad 2017} & Metformin & 104.4(13.12)* & 93.48(11.9) & $-10.92(12.55)$ & NA & NA & NA & $5.28(0.42)$ \\
\hline & Insulin & $117.9(29.06) *$ & $102.08(20.63)$ & $-15.82(25.9)$ & NA & NA & NA & $5.43(0.34)$ \\
\hline \multirow[t]{2}{*}{ Gamal 2018} & Metformin & NA & NA & NA & NA & NA & NA & $6.9(0.4)$ \\
\hline & Insulin & NA & NA & NA & NA & NA & NA & $6.7(0.5)$ \\
\hline \multirow[t]{2}{*}{ Wasim 2019} & Metformin & 117(18.1) & $92.1(6)$ & $-24.9(15.97)$ & NA & NA & NA & $6.99(7.5)$ \\
\hline & Insulin & $120(22.4)$ & $96.6(6.2)$ & $-23.4(20.03)$ & NA & NA & NA & 7.1(0.79) \\
\hline \multirow[t]{2}{*}{ Langer 2000} & Glyburide & 104(25) & $98(13)$ & $-6(21.7)$ & 174(31) & 113(22) & $-61(27.6)$ & $5.7(1.3)$ \\
\hline & Insulin & 108(26) & $98(16)$ & $-10(22.7)$ & 174(39) & 112(15) & $-62(34.1)$ & $5.6(1.2)$ \\
\hline \multirow{2}{*}{$\begin{array}{l}\text { Anjalakshi } \\
2007\end{array}$} & Glyburide & NA & NA & NA & 167.1(22.97) & $95.29(7.41)$ & $-71.81(20.31)$ & $5.48(0.79)$ \\
\hline & Insulin & NA & NA & NA & 174.92(31.05) & $93(9.75)$ & $-81.92(27.5)$ & $5.75(1.23)$ \\
\hline \multirow{2}{*}{$\begin{array}{l}\text { Ogunyemi } \\
2007\end{array}$} & Glyburide & 103.7(20.1)* & $95.6(13.4)$ & $-8.1(17.73)$ & 179(34.7) & $132.6(35)$ & $-46.4(34.9)$ & $5.8(0.7) *$ \\
\hline & Insulin & 115.8(19.6)* & $89.8(13.2)$ & $-26(17.31)$ & 197.1(42) & 116.3(16.6) & $-80.8(36.6)$ & 7.5(1.7)* \\
\hline \multirow[t]{2}{*}{ Lain 2009} & Glyburide & 100.9(15.9) & $90.4(21.8)$ & $-10.5(19.53)$ & 176.9(35.9) & 109.8(21.8) & $-67.1(31.33)$ & $5(0.5)$ \\
\hline & Insulin & 101.5(12.4) & $90.9(7)$ & $-10.6(10.77)$ & 173.1(34.9) & 106(14) & $-67.1(30.42)$ & $5(0.5)$ \\
\hline \multirow{2}{*}{$\begin{array}{l}\text { Mukhopadhyay } \\
2012\end{array}$} & Glyburide & $103.5(14.62)$ & $88.23(6.55)$ & $-15.27(12.68)$ & $184.1(20.46)$ * & $122.7(10.3)$ & $-61.4(17.72)$ & $6.25(0.6)$ \\
\hline & Insulin & 109.3(19.63) & $88.17(8.44)$ & $-21.13(17.06)$ & $194.3(18.47) *$ & $128(12.38)$ & $-66.3(16.3)$ & $6.46(0.77)$ \\
\hline \multirow{2}{*}{$\begin{array}{l}\text { Mirzamoradi } \\
2015\end{array}$} & Glyburide & 109.83(68.99) & $114.02(10.65)$ & $4.19(64.33)$ & NA & 115.46(8.21) & NA & NA \\
\hline & Insulin & 112.15(19.39) & $123.42(14.71)$ & 11.27(17.53) & NA & $120.15(9.56)$ & NA & NA \\
\hline \multirow[t]{2}{*}{ Silva 2012} & Glyburide & $94.04(16.25)$ & $88.23(11.71)$ & $-5.81(14.52)$ & 160.83(18.6) & $126.44(16.91)$ & $-34.39(17.81)$ & NA \\
\hline & Metformin & 95.84(20.91) & $90.52(11.78)$ & $-5.32(18.16)$ & 165.59(21.9) & 126.48(20.51) & $-39.11(21.18)$ & NA \\
\hline \multirow[t]{2}{*}{ George 2015} & Glyburide & $100.8(14.4)$ & $86.4(24.4)$ & $-14.4(21.24)$ & $181.8(45)$ & $119.4(21.25)$ & $-62.4(38.99)$ & $5.9(0.5)$ \\
\hline & Metformin & $102.6(14.4)$ & $88.2(10.8)$ & $-14.4(12.98)$ & $194.4(46.8)$ & 123.6(15.41) & $-70.8(41.31)$ & $5.8(0.6)$ \\
\hline
\end{tabular}




\begin{tabular}{|c|c|c|c|c|c|c|c|c|}
\hline \multirow[t]{2}{*}{ Nachum 2017} & Glyburide & $95.9(10.4)$ & $88.7(10.2)$ & $-7.2(10.3)$ & 127.6(19.1) & 115.3(13.8) & $-12.3(17.08)$ & NA \\
\hline & Metformin & $96.8(10.5)$ & 91.3(8.8) & $-5.5(9.76)$ & $125.4(12.8)$ & $112.6(12.3)$ & $-12.8(12.56)$ & NA \\
\hline
\end{tabular}

Table 3

Network meta-analysis of glycemic control and birth weight.

\begin{tabular}{|c|c|c|c|c|c|c|c|c|c|c|c|}
\hline FBG & & & 2HPG & & & HbA1c & & & Birth weight & & \\
\hline Glyburide & & & Glyburid & & & Glyburide & & & Glyburide & & \\
\hline 5.48 & \multirow[t]{2}{*}{ Insulin } & & 1.91 & \multirow[t]{2}{*}{ Insulin } & & 0.01 & \multirow[t]{2}{*}{ Insulin } & & 0.05 & \multirow[t]{2}{*}{ Insulin } & \\
\hline$(-8.58,19.85)$ & & & $\begin{array}{l}(-4.34 \\
8.59)\end{array}$ & & & $\begin{array}{l}(-0.18 \\
0.22)\end{array}$ & & & $(-0.03,0.14)$ & & \\
\hline-2.74 & -8.18 & \multirow[t]{2}{*}{ Metformin } & 5.08 & 3.23 & \multirow[t]{2}{*}{ Metformin } & 0.01 & 0.00 & \multirow[t]{2}{*}{ Metformin } & 0.13 & 0.09 & \multirow[t]{2}{*}{ Metfo } \\
\hline$(-17.17,11.23)$ & $(-19.30,2.46)$ & & $\begin{array}{l}(-1.18 \\
11.76)\end{array}$ & $\begin{array}{l}(-2.39 \\
8.73)\end{array}$ & & $\begin{array}{l}(-0.19, \\
0.23)\end{array}$ & $\begin{array}{l}(-0.07 \\
0.08)\end{array}$ & & $(0.05,0.23)$ & $(0.02,0.16)$ & \\
\hline
\end{tabular}

Table 4

Probability of ranking by treatment.

\begin{tabular}{|c|c|c|c|c|c|c|c|c|c|c|}
\hline & & \multicolumn{3}{|c|}{ Glyburide } & \multicolumn{3}{|c|}{ Metformin } & \multicolumn{3}{|l|}{ Insulin } \\
\hline & & \multicolumn{3}{|c|}{ Worst Best } & \multicolumn{3}{|c|}{ Worst Best } & \multicolumn{3}{|c|}{ Worst Best } \\
\hline & & Rank1 & Rank2 & Rank3 & Rank1 & Rank2 & Rank3 & Rank1 & Rank2 & Rank3 \\
\hline \multirow[t]{3}{*}{ Glycemic control } & FBG & 0.33 & 0.47 & 0.20 & 0.64 & 0.32 & 0.04 & 0.3 & 0.21 & 0.76 \\
\hline & $2 \mathrm{HPG}$ & 0.72 & 0.24 & 0.04 & 0.02 & 0.12 & 0.86 & 0.26 & 0.64 & 0.10 \\
\hline & HbA1c & 0.54 & 0.10 & 0.36 & 0.24 & 0.42 & 0.34 & 0.22 & 0.48 & 0.30 \\
\hline \multirow[t]{12}{*}{ Neonatal outcomes } & Anomaly & 0.78 & 0.10 & 0.12 & 0.11 & 0.31 & 0.58 & 0.11 & 0.59 & 0.30 \\
\hline & Hyperbiliru & 0.82 & 0.12 & 0.06 & 0.02 & 0.21 & 0.76 & 0.16 & 0.67 & 0.17 \\
\hline & LGA & 0.94 & 0.05 & 0.01 & 0.01 & 0.18 & 0.80 & 0.05 & 0.77 & 0.19 \\
\hline & Macrosomia & 0.96 & 0.04 & 0.00 & 0.00 & 0.05 & 0.95 & 0.04 & 0.91 & 0.05 \\
\hline & NICU admission & 0.23 & 0.38 & 0.38 & 0.04 & 0.36 & 0.60 & 0.73 & 0.26 & 0.02 \\
\hline & Neonatal hypoglycemia & 0.95 & 0.05 & 0.00 & 0.00 & 0.02 & 0.97 & 0.05 & 0.93 & 0.02 \\
\hline & Obstetric trauma & 0.38 & 0.26 & 0.36 & 0.17 & 0.28 & 0.54 & 0.45 & 0.46 & 0.09 \\
\hline & Perinatal death & 0.56 & 0.27 & 0.17 & 0.11 & 0.22 & 0.67 & 0.33 & 0.50 & 0.16 \\
\hline & Preterm birth & 0.35 & 0.35 & 0.30 & 0.58 & 0.35 & 0.07 & 0.07 & 0.30 & 0.63 \\
\hline & RDS & 0.28 & 0.21 & 0.51 & 0.30 & 0.36 & 0.34 & 0.41 & 0.43 & 0.15 \\
\hline & SGA & 0.42 & 0.11 & 0.47 & 0.48 & 0.39 & 0.12 & 0.10 & 0.50 & 0.41 \\
\hline & Birth weight & 0.88 & 0.12 & 0.00 & 0.00 & 0.01 & 0.99 & 0.12 & 0.87 & 0.01 \\
\hline Preeclampsia & & 0.64 & 0.27 & 0.09 & 0.01 & 0.10 & 0.89 & 0.35 & 0.63 & 0.02 \\
\hline
\end{tabular}

\subsection{HbA1c}

Pairwise meta-analysis of the change in $\mathrm{HbA} 1 \mathrm{c}$ from baseline showed no significance differences between metformin and insulin, or glyburide and insulin (Supplementary Fig. 6). The NWA revealed no significant differences between the change in HbA1c from baseline associated with each treatment (Table 3). Glyburide had the highest probability of being the least effective treatment (54\%) (Table 4).

\subsection{Neonatal outcomes}

\subsubsection{Neonatal hypoglycemia}

Pairwise meta-analysis showed a significant lower prevalence of neonatal hypoglycemia in the metformin group than in the insulin group (RD - $0.07 ; 95 \% \mathrm{Cl}-$ 0.11--0.03) (Table 5). Moreover, there was higher prevalence in the glyburide group than in the insulin group (RD 0.05, 95\% Cl 0.02-0.08). However, no significant difference was found between the glyburide and metformin groups, which may be because of the heterogeneity in the trials $\left(I^{2}, 82 \%\right)$. Metformin had the highest probability of being the best option (97\%), while the glyburide had the highest probability of being the worst option (95\%), and insulin had the highest probability of ranking second (93\%) (Table 4).

\subsubsection{NICU admission}

Pairwise meta-analysis showed a significantly lower prevalence of NICU admission in the metformin group than in the insulin group (RD - 0.03 ; $95 \% \mathrm{Cl}-$ 0.06-0) (Table 5). However, no significant differences were found between glyburide and insulin or glyburide and metformin. Metformin had the highest 
probability of being the best option (60\%), while insulin had the highest probability of being the worst option (73\%) (Table 4).

\subsubsection{Macrosomia}

Pairwise meta-analysis of macrosomia showed a lower prevalence in the metformin group than in the insulin group (RD - 0.03; $95 \% \mathrm{Cl}-0.06-0)$, but the difference was not significant ( $p=0.09$ ) (Table 5). Furthermore, the prevalence of macrosomia was higher in the glyburide group than in the insulin group (RD $0.04 ; 95 \% \mathrm{Cl} 0-0.08)$, but the difference was not significant $(p=0.07)$. No significant difference was found between the glyburide and metformin groups. In the NMA, metformin was the best option, with a probability of $95 \%$, while glyburide had the highest probability of being the worst option ( $96 \%$ ). Insulin had the highest probability of ranking second (91\%) (Table 4).

\subsubsection{Birth weight}

Supplementary Fig. 7 shows the results of the pairwise meta-analysis of birth weight. The metformin group showed significantly lower birth weight than the insulin group (SMD - 0.25; 95\% Cl - 0.41--0.10) and the glyburide group showed significantly higher birth weight than the insulin group (SMD $0.10 ; 95 \% \mathrm{Cl}$ $0.01-0.20)$. The pairwise meta-analysis of glyburide and metformin showed high heterogeneity $\left(I^{2}, 72 \%\right)$ and no significant difference was detected. The NWA results were consistent with those of the pairwise meta-analysis (Table 3). Metformin had the highest probability of being the best option for the control of birth weight (99\%), while glyburide had the highest probability of being the worst option (88\%). Insulin had the highest probability of ranking second (87\%) (Table 4).

\subsubsection{Other neonatal outcomes}

No significant differences were identified in the pairwise meta-analyses of anomalies, neonatal hyperbilirubinemia, obstetric trauma, perinatal death, preterm birth, RDS, LGA, or SGA (Table 5).

\subsection{Maternal outcomes}

\subsubsection{Preeclampsia}

Pairwise meta-analysis of PE showed significantly lower prevalence in the metformin group than in the insulin group (RD - 0.03; $95 \% \mathrm{Cl}-0.06-0)$ (Table 5). However, no significant differences were found between glyburide and insulin or glyburide and metformin. The NMA revealed that metformin had the highest probability of being the most effective treatment for the prevention of PE (89\%), while glyburide had the highest probability of being the least effective treatment (64\%). Insulin had the highest probability of ranking second (63\%) (Table 4).

\subsubsection{Maternal weight gain}

The only studies of maternal weight gain had compared metformin with insulin. Therefore, no NMA was conducted. The pairwise meta-analysis showed lower maternal weight gain after enrollment in the metformin group than in the insulin group (SMD $-0.61 ; 95 \% \mathrm{Cl}-0.86--0.35)$ (Supplementary Fig. 8).

\section{Discussions}

The present network meta-analysis included 24 comparisons of 4,683 individuals with GDM who had been randomly assigned to glyburide, metformin, or insulin treatment. The results demonstrate that metformin is as effective as insulin for glycemic control, and is the most promising drug for the prevention of neonatal and maternal complications among the treatments compared. However, glyburide seems to be inferior to both insulin and metformin with respect to glycemic control, which may also explain the poorer neonatal outcomes that were associated with the use of this drug.

Metformin seems to be better than glyburide and no worse than insulin with respect to glycemic control. Moreover, metformin had the highest probability of being the most effective drug for the control of $2 \mathrm{HPG}$ among the three treatments evaluated, according to the NMA. This finding is consistent with the mechanisms of action of metformin, which increases insulin sensitivity and glucose uptake into muscle, liver, and adipose tissue [64]. Glyburide was significantly less effective at reducing FBG than insulin and 2HPG than metformin. However, no significant difference was found for the control of HbA1c.

The metformin group had lower prevalences of neonatal hypoglycemia, macrosomia, and NICU admission, while the glyburide group had a higher prevalence of neonatal hypoglycemia than insulin. This result was consistent with the effects of the drugs on glycemic control, and suggests that maternal blood glucose control, and especially 2HPG control, may be an important predictor of the neonatal outcomes of hypoglycemia, macrosomia, and NICU admission. However, no significant differences were found in the prevalences of severe neonatal conditions, such as anomalies, perinatal death, or RDS, between the use of OHAs and insulin. NMA showed that metformin had the highest probability of being the safest treatment for the prevention of the neonatal complications of macrosomia (95\%), neonatal hypoglycemia (97\%), LGA (80\%), neonatal hyperbilirubinemia (76\%), perinatal death (67\%), NICU admission (60\%), and anomalies (58\%). This suggests that metformin is a safe treatment with respect to short-term neonatal outcomes.

Regarding the long-term outcomes, previous studies have shown that GDM is associated with obesity and diabetes in offspring [65-69]. Intrauterine hyperglycemia can result in epigenetic and structural alterations of fetal tissues, which are proposed mechanisms for the fetal origin of adult diseases. Thus, better glycemic control is thought to be associated with superior long-term outcomes. However, because both of the OHAs are thought to pass across the placenta, the direct effects of metformin and glyburide on fetal development require investigation. As stated above, the metformin group had significant lower birth weight and a lower prevalence of macrosomia than the insulin group. ljas et al. reported no significant difference in the ponderal index between the 18month-old children of metformin- and insulin-treated mothers. Moreover, they also showed that the children of metformin-treated mothers were taller and heavier than those of insulin-treated mothers [34]. Rowan et al. reported larger amounts of subcutaneous fat in the 2-year-old children of metformin-treated 
mothers than in the children of insulin-treated mothers [36]. Furthermore, Wouldes et al. found that the children of metformin-treated mothers had similar neurodevelopmental outcomes to those of insulin-treated mothers at 2 years of age [35]. However, because of the limited number of studies conducted and the differing follow-up periods, it is not feasible to carry out meta-analyses of these outcomes. Moreover, studies with a longer follow-up period are required because some of the diseases that have fetal origins have onsets in early adulthood or at an even later stage.

Recent meta-analyses studies have compared the safety and efficiency of OHAs and insulin [29,30]. However, no significant differences were identified in the glycemic control achieved using OHAs and insulin, at least in part due to high heterogeneity among the included trials. In the present meta-analysis, the heterogeneity among the studies was lower than in previous analyses. The present study design was different to that of previous meta-analyses in two principal respects. First, the changes from baseline in FBG, 2HPG, HbA1c during treatment were selected as the primary outcomes, rather than the FBG, $2 \mathrm{HPG}$, $\mathrm{HbA} 1 \mathrm{c}$ levels after treatment. In this way, the effects of differences in baseline blood glucose levels between populations were reduced. Second, studies that used a PP protocol and those with significantly different baseline maternal ages and gestational ages at enrollment were excluded.

Only ITT studies were included in the pairwise and network meta-analysis. The studies that used a PP protocol excluded patients with inadequate glycemic control, which meant that patients with more serious disease were excluded, which would likely have resulted in differing baseline values between the two groups and inconsistencies when data from these studies were pooled with those from ITT studies. However, although there may have been statistical inconsistencies between the PP and ITT studies, the conclusions of the present meta-analysis were consistent with those of most of the PP studies. Mesdaghinia et al. reported a lower prevalence of macrosomia in metformin-treated mothers than insulin-treated mothers, and the prevalences of LGA, neonatal hyperbilirubinemia, RDS, and anomalies tended to be lower in the metformin group, although significant differences were not identified [46]. Ghomian et al. showed no significant differences in glycemic control, birth weight, or the prevalences of preterm birth or cesarean section between the metformin and insulin groups [62]. The prevalence of neonatal hypoglycemia tended to be lower in the metformin group, although this did not achieve significance [62]. Ruholamin et al. reported no significant differences in the prevalences of gestational complications, preterm birth, hyperbilirubinemia, NICU admission, macrosomia, SGA, or neonatal hypoglycemia between the metformin and insulin groups. However, the neonatal blood glucose concentrations $1 \mathrm{~h}$ and $2 \mathrm{~h}$ after birth were significantly lower in the insulin group than in the metformin group [52]. Finally, Behrashi et al. compared glyburide and insulin treatment and found that the glyburide group had a lower prevalence of macrosomia and lower birth weight than the insulin group. However, there were no differences in the prevalences of neonatal hypoglycemia, RDS, hyperbilirubinemia, anomalies, or NICU admission [57].

Two studies were excluded because of poor matching of the groups at baseline. Because their sample sizes were relatively small, significant differences at baseline can confound assessments of the severity of the disease or lead to different treatment durations in the two groups, such that the comparisons would not be effectively controlled. The study reported by Ogunymi et al. was characterized by significantly different gestational ages at enrollment (metformin vs. insulin, $28.1 \pm 7.6$ weeks vs. $24.6 \pm 8$ weeks), and therefore this study was not included in the meta-analysis to avoid introducing bias, despite the fact that they reported similar results to those of the present study with respect to the effects of insulin and glyburide on $2 \mathrm{HPG}$ control and the prevalence of neonatal hypoglycemia.[42] Similarly, the study reported by Mirzamoradi et al. was not included because of significantly different maternal age in the glyburide and insulin groups.[55]

The prevalence of treatment failure was also compared between the glyburide and metformin groups, but no significant difference was found (Supplementary Fig. 9). However, because the two drugs regulate blood glucose through different mechanisms, glyburide may be effective in patients who do not respond to metformin. Thus, glyburide may represent an alternative treatment for patients who are not willing to administer daily injections and frequently monitor their blood glucose.

Almost all of the included studies were conducted in urban areas. However, patients in rural areas may be more likely to have a lower educational level and be less affluent, which can result in non-compliance and inadequate glucose monitoring during insulin treatment. Therefore, the effectiveness of insulin treatment may be overestimated for patients in rural areas. To provide complete evidence for decision makers, the cost-benefit ratio is a very important index of the utility of a therapy. However, the mean dosage of drug administered and their costs were missing from most of the studies. Moreover, because of the different methods of administration of OHAs and insulin, it is impossible to blind the patients, and therefore all the studies comparing the effects of OHA and insulin had a risk of bias. Last but not least, most of the studies were conducted with modest sample sizes; therefore, differences between groups may have been missed due to a lack of power.

In conclusion, the present study provides evidence that metformin is as effective as insulin for glycemic control in GDM and is superior with respect to the prevention of adverse neonatal outcomes, such as neonatal hypoglycemia, macrosomia, and NICU admission. However, further studies with larger sample sizes are required to assess the long-term effects of metformin treatment on offspring outcomes and to determine the cost-benefit ratios of the use of the drugs in rural populations.

\section{Declarations}

\section{Consent for publication}

Written informed consent for publication was obtained from all participants.

\section{Availability of data and materials}

All data generated or analysed during this study are included in this published article and its supplementary information files.

\section{Acknowledgments}

Page 9/12 
We thank Mark Cleasby, PhD, from Liwen Bianji, Edanz Group China (www.liwenbianji.cn/ac), for editing the English text of a draft of this manuscript.

\section{Funding}

This work was supported by the Zhejiang Provincial Natural Science Foundation of China (Grants LY19H040014 to Li-Quan Wang) and National Natural Science Foundation of China (Grants 81901498 to Dan-Qing Yu and 81871176 to Chun Feng).

\section{Competing interests}

The authors have no conflicts of interest to disclose.

\section{Authors' contributions}

L.W. and D. Y. designed the study. G. X. and D. Y. did the literature search, data collection and wrote the main manuscript text. J. X. and X. T. independently assessed the risk of bias. L. T. and C. F. prepared table 1-2. J. R. and M. J. prepared table 3-5. All authors reviewed the manuscript. D. Y. and G. X. contributed equally to this work and should be considered joint first authors.

\section{References}

1. Ben-Haroush A, Yogev Y, Hod M. Epidemiology of gestational diabetes mellitus and its association with Type 2 diabetes. Diabet Med. 2004;21:103-13.

2. Ferrara A, Kahn HS, Quesenberry CP, Riley C, Hedderson MM. An increase in the incidence of gestational diabetes mellitus: Northern California, 19912000. Obstet Gynecol. 2004;103:526-33.

3. Guariguata L, Whiting DR, Hambleton I, Beagley J, Linnenkamp U, Shaw JE. Global estimates of diabetes prevalence for 2013 and projections for 2035. Diabetes Res Clin Pract. 2014;103:137-49.

4. Ferrara A. Increasing prevalence of gestational diabetes mellitus: a public health perspective. Diabetes Care. 2007;30 Suppl 2:S141-6.

5. DeSisto CL, Kim SY, Sharma AJ. Prevalence estimates of gestational diabetes mellitus in the United States, Pregnancy Risk Assessment Monitoring System (PRAMS), 2007-2010. Prev Chronic Dis. 2014;11:E104.

6. Baptiste-Roberts K, Barone BB, Gary TL, Golden SH, Wilson LM, Bass EB, et al. Risk factors for type 2 diabetes among women with gestational diabetes: a systematic review. Am J Med. 2009;122:207 - 14 e4.

7. International Association of D, Pregnancy Study Groups Consensus P, Metzger BE, Gabbe SG, Persson B, Buchanan TA, et al. International association of diabetes and pregnancy study groups recommendations on the diagnosis and classification of hyperglycemia in pregnancy. Diabetes Care. 2010;33:67682.

8. Esakoff TF, Cheng YW, Sparks TN, Caughey AB. The association between birthweight $4000 \mathrm{~g}$ or greater and perinatal outcomes in patients with and without gestational diabetes mellitus. Am J Obstet Gynecol. 2009;200:672 e1-4.

9. Langer O. A spectrum of glucose thresholds may effectively prevent complications in the pregnant diabetic patient. Semin Perinatol. 2002;26:196-205.

10. Greger HK, Hanem LGE, Ostgard HF, Vanky E. Cognitive function in metformin exposed children, born to mothers with PCOS - follow-up of an RCT. BMC Pediatr. 2020;20:60.

11. Bidhendi Yarandi R, Behboudi-Gandevani S, Amiri M, Ramezani Tehrani F. Metformin therapy before conception versus throughout the pregnancy and risk of gestational diabetes mellitus in women with polycystic ovary syndrome: a systemic review, meta-analysis and meta-regression. Diabetol Metab Syndr. 2019;11:58.

12. Committee on Practice B-O. ACOG Practice Bulletin No. 190: Gestational Diabetes Mellitus. Obstet Gynecol. 2018;131:e49-e64.

13. Simmons D, McElduff A, Mclntyre HD, Elrishi M. Gestational diabetes mellitus: NICE for the U.S.? A comparison of the American Diabetes Association and the American College of Obstetricians and Gynecologists guidelines with the U.K. National Institute for Health and Clinical Excellence guidelines. Diabetes Care. 2010;33:34-7.

14. Rai L, Meenakshi D, Kamath A. Metformin-a convenient alternative to insulin for Indian women with diabetes in pregnancy. Indian J Med Sci. 2009;63:491-7.

15. Schwartz RA, Rosenn B, Aleksa K, Koren G. Glyburide transport across the human placenta. Obstet Gynecol. 2015;125:583-8.

16. Hebert MF, Ma X, Naraharisetti SB, Krudys KM, Umans JG, Hankins GD, et al. Are we optimizing gestational diabetes treatment with glyburide? The pharmacologic basis for better clinical practice. Clin Pharmacol Ther. 2009;85:607-14.

17. Balsells M, Garcia-Patterson A, Sola I, Roque M, Gich I, Corcoy R. Glibenclamide, metformin, and insulin for the treatment of gestational diabetes: a systematic review and meta-analysis. BMJ. 2015;350:h102.

18. Moretti ME, Rezvani M, Koren G. Safety of glyburide for gestational diabetes: a meta-analysis of pregnancy outcomes. Ann Pharmacother. 2008;42:48390.

19. Cheng YW, Chung JH, Block-Kurbisch I, Inturrisi M, Caughey AB. Treatment of gestational diabetes mellitus: glyburide compared to subcutaneous insulin therapy and associated perinatal outcomes. J Matern Fetal Neonatal Med. 2012;25:379-84.

20. Poolsup N, Suksomboon N, Amin M. Efficacy and safety of oral antidiabetic drugs in comparison to insulin in treating gestational diabetes mellitus: a meta-analysis. PLoS One. 2014;9:e109985.

21. Zeng YC, Li MJ, Chen Y, Jiang L, Wang SM, Mo XL, et al. The use of glyburide in the management of gestational diabetes mellitus: a meta-analysis. Adv Med Sci. 2014;59:95-101. 
22. Camelo Castillo W, Boggess K, Sturmer T, Brookhart MA, Benjamin DK, Jr., Jonsson Funk M. Association of Adverse Pregnancy Outcomes With Glyburide vs Insulin in Women With Gestational Diabetes. JAMA Pediatr. 2015;169:452-8.

23. Jiang YF, Chen XY, Ding T, Wang XF, Zhu ZN, Su SW. Comparative efficacy and safety of OADs in management of GDM: network meta-analysis of randomized controlled trials. J Clin Endocrinol Metab. 2015;100:2071-80.

24. Song R, Chen L, Chen Y, Si X, Liu Y, Liu Y, et al. Comparison of glyburide and insulin in the management of gestational diabetes: A meta-analysis. PLoS One. 2017;12:e0182488.

25. Niromanesh S, Alavi A, Sharbaf FR, Amjadi N, Moosavi S, Akbari S. Metformin compared with insulin in the management of gestational diabetes mellitus: a randomized clinical trial. Diabetes Res Clin Pract. 2012;98:422-9.

26. Rowan JA, Hague WM, Gao W, Battin MR, Moore MP, Mi GTI. Metformin versus insulin for the treatment of gestational diabetes. N Engl J Med. 2008;358:2003-15.

27. Hellmuth E, Damm P, Molsted-Pedersen L. Oral hypoglycaemic agents in 118 diabetic pregnancies. Diabet Med. 2000;17:507-11.

28. Silva JC, Fachin DR, Coral ML, Bertini AM. Perinatal impact of the use of metformin and glyburide for the treatment of gestational diabetes mellitus. $J$ Perinat Med. 2012;40:225-8.

29. Bao LX, Shi WT, Han YX. Metformin versus insulin for gestational diabetes: a systematic review and meta-analysis. J Matern Fetal Neonatal Med. 2019:1-13.

30. Guo L, Ma J, Tang J, Hu D, Zhang W, Zhao X. Comparative Efficacy and Safety of Metformin, Glyburide, and Insulin in Treating Gestational Diabetes Mellitus: A Meta-Analysis. J Diabetes Res. 2019;2019:9804708.

31. Scott DA, Boye KS, Timlin L, Clark JF, Best JH. A network meta-analysis to compare glycaemic control in patients with type 2 diabetes treated with exenatide once weekly or liraglutide once daily in comparison with insulin glargine, exenatide twice daily or placebo. Diabetes Obes Metab. 2013;15:21323.

32. Ma J, Liu W, Hunter A, Zhang W. Performing meta-analysis with incomplete statistical information in clinical trials. BMC Med Res Methodol. $2008 ; 8: 56$.

33. Follmann D, Elliott P, Suh I, Cutler J. Variance imputation for overviews of clinical trials with continuous response. J Clin Epidemiol. 1992;45:769-73.

34. ljas H, Vaarasmaki M, Saarela T, Keravuo R, Raudaskoski T. A follow-up of a randomised study of metformin and insulin in gestational diabetes mellitus: growth and development of the children at the age of 18 months. BJOG. 2015;122:994-1000.

35. Wouldes TA, Battin M, Coat S, Rush EC, Hague WM, Rowan JA. Neurodevelopmental outcome at 2 years in offspring of women randomised to metformin or insulin treatment for gestational diabetes. Arch Dis Child Fetal Neonatal Ed. 2016;101:F488-F93.

36. Rowan JA, Rush EC, Obolonkin V, Battin M, Wouldes T, Hague WM. Metformin in gestational diabetes: the offspring follow-up (MiG TOFU): body composition at 2 years of age. Diabetes Care. 2011;34:2279-84.

37. Langer O, Conway DL, Berkus MD, Xenakis EM, Gonzales O. A comparison of glyburide and insulin in women with gestational diabetes mellitus. N Engl J Med. 2000;343:1134-8.

38. Hague WM, Davoren PM, Oliver J, Rowan J. Contraindications to use of metformin. Metformin may be useful in gestational diabetes. BMJ. 2003;326:762; author reply

39. Bertini AM, Silva JC, Taborda W, Becker F, Lemos Bebber FR, Zucco Viesi JM, et al. Perinatal outcomes and the use of oral hypoglycemic agents. J Perinat Med. 2005;33:519-23.

40. Anjalakshi C, Balaji V, Balaji MS, Seshiah V. A prospective study comparing insulin and glibenclamide in gestational diabetes mellitus in Asian Indian women. Diabetes Res Clin Pract. 2007;76:474-5.

41. Moore LE, Briery CM, Clokey D, Martin RW, Williford NJ, Bofill JA, et al. Metformin and insulin in the management of gestational diabetes mellitus: preliminary results of a comparison. J Reprod Med. 2007;52:1011-5.

42. Ogunyemi D, Jesse M, Davidson M. Comparison of glyburide versus insulin in management of gestational diabetes mellitus. Endocr Pract. 2007;13:4278.

43. Lain KY, Garabedian MJ, Daftary A, Jeyabalan A. Neonatal adiposity following maternal treatment of gestational diabetes with glyburide compared with insulin. Am J Obstet Gynecol. 2009;200:501 e1-6.

44. Moore LE, Clokey D, Rappaport VJ, Curet LB. Metformin compared with glyburide in gestational diabetes: a randomized controlled trial. Obstet Gynecol. 2010;115:55-9.

45. ljas H, Vaarasmaki M, Morin-Papunen L, Keravuo R, Ebeling T, Saarela T, et al. Metformin should be considered in the treatment of gestational diabetes: a prospective randomised study. BJOG. 2011;118:880-5.

46. Mesdaghinia E, Samimi M, Homaei Z, Saberi F, Moosavi SG, Yaribakht M. Comparison of newborn outcomes in women with gestational diabetes mellitus treated with metformin or insulin: a randomised blinded trial. Int J Prev Med. 2013;4:327-33.

47. Hassan JA, Karim N, Sheikh Z. Metformin prevents macrosomia and neonatal morbidity in gestational diabetes. Pakistan Journal of Medical Sciences. 2012;28:384-9.

48. Mukhopadhyay P, Bag TS, Kyal A, Saha DP, Khalid N. Oral hypoglycemic glibenclamide: Can it be a substitute to insulin in the management of gestational diabetes mellitus? a comparative study. Journal of SAFOG. 2012;4:28-31.

49. Spaulonci CP, Bernardes LS, Trindade TC, Zugaib M, Francisco RP. Randomized trial of metformin vs insulin in the management of gestational diabetes. Am J Obstet Gynecol. 2013;209:34 e1-7.

50. Tempe A, Mayanglambam RD. Glyburide as treatment option for gestational diabetes mellitus. J Obstet Gynaecol Res. 2013;39:1147-52.

Page $11 / 12$ 
51. Tertti K, Ekblad U, Koskinen P, Vahlberg T, Ronnemaa T. Metformin vs. insulin in gestational diabetes. A randomized study characterizing metformin patients needing additional insulin. Diabetes Obes Metab. 2013;15:246-51.

52. Ruholamin S, Eshaghian S, Allame Z. Neonatal outcomes in women with gestational diabetes mellitus treated with metformin in compare with insulin: A randomized clinical trial. J Res Med Sci. 2014;19:970-5.

53. Ainuddin J, Karim N, Hasan AA, Naqvi SA. Metformin versus insulin treatment in gestational diabetes in pregnancy in a developing country: a randomized control trial. Diabetes Res Clin Pract. 2015;107:290-9.

54. George A, Mathews JE, Sam D, Beck M, Benjamin SJ, Abraham A, et al. Comparison of neonatal outcomes in women with gestational diabetes with moderate hyperglycaemia on metformin or glibenclamide-a randomised controlled trial. Aust N Z J Obstet Gynaecol. 2015;55:47-52.

55. Mirzamoradi M, Heidar Z, Faalpoor Z, Naeiji Z, Jamali R. Comparison of glyburide and insulin in women with gestational diabetes mellitus and associated perinatal outcome: a randomized clinical trial. Acta Med Iran. 2015;53:97-103.

56. Ashoush S, El-Said M, Fathi H, Abdelnaby M. Identification of metformin poor responders, requiring supplemental insulin, during randomization of metformin versus insulin for the control of gestational diabetes mellitus. J Obstet Gynaecol Res. 2016;42:640-7.

57. Behrashi M, Samimi M, Ghasemi T, Saberi F, Atoof F. Comparison of Glibenclamide and Insulin on Neonatal Outcomes in Pregnant Women with Gestational Diabetes. Int J Prev Med. 2016;7:88.

58. Saleh HS, Abdelsalam WA, Mowafy HE, Abd ElHameid AA. Could Metformin Manage Gestational Diabetes Mellitus instead of Insulin? Int J Reprod Med. 2016;2016:3480629.

59. Arshad R, Khanam S, Shaikh F, Karim N. Feto-maternal outcomes and Glycemic control in Metformin versus insulin treated Gestational Diabetics. Pak J Med Sci. 2017;33:1182-7.

60. Nachum Z, Zafran N, Salim R, Hissin N, Hasanein J, Gam Ze Letova Y, et al. Glyburide Versus Metformin and Their Combination for the Treatment of Gestational Diabetes Mellitus: A Randomized Controlled Study. Diabetes Care. 2017;40:332-7.

61. Senat MV, Affres H, Letourneau A, Coustols-Valat M, Cazaubiel M, Legardeur H, et al. Effect of Glyburide vs Subcutaneous Insulin on Perinatal Complications Among Women With Gestational Diabetes: A Randomized Clinical Trial. JAMA. 2018;319:1773-80.

62. Ghomian N, Vahed SHM, Firouz S, Yaghoubi MA, Mohebbi M, Sahebkar A. The efficacy of metformin compared with insulin in regulating blood glucose levels during gestational diabetes mellitus: A randomized clinical trial. J Cell Physiol. 2019;234:4695-701.

63. Wasim T, Shaukat S, Javaid L, Mukhtar S, Amer W. Comparison of Metformin and Insulin for Management of Gestational Diabetes Mellitus: A Randomized Control Trial. Pakistan Journal of Medical \& Health Sciences. 2019;13:823-7.

64. Rena G, Pearson ER, Sakamoto K. Molecular mechanism of action of metformin: old or new insights? Diabetologia. 2013;56:1898-906.

65. Petitt DJ, Bennett PH, Knowler WC, Baird HR, Aleck KA. Gestational diabetes mellitus and impaired glucose tolerance during pregnancy. Long-term effects on obesity and glucose tolerance in the offspring. Diabetes. 1985;34 Suppl 2:119-22.

66. Group HSCR, Metzger BE, Lowe LP, Dyer AR, Trimble ER, Chaovarindr U, et al. Hyperglycemia and adverse pregnancy outcomes. N Engl J Med. 2008;358:1991-2002.

67. Clausen TD, Mathiesen ER, Hansen T, Pedersen O, Jensen DM, Lauenborg J, et al. Overweight and the metabolic syndrome in adult offspring of women with diet-treated gestational diabetes mellitus or type 1 diabetes. J Clin Endocrinol Metab. 2009;94:2464-70.

68. Nehring I, Chmitorz A, Reulen H, von Kries R, Ensenauer R. Gestational diabetes predicts the risk of childhood overweight and abdominal circumference independent of maternal obesity. Diabet Med. 2013;30:1449-56.

69. Nilsson C, Carlsson A, Landin-Olsson M. Increased risk for overweight among Swedish children born to mothers with gestational diabetes mellitus. Pediatr Diabetes. 2014;15:57-66.

\section{Tables}

Due to technical limitations, table 5 is only available as a download in the Supplemental Files section.

\section{Supplementary Files}

Supplementary files not available with this version.

\section{Supplementary Files}

This is a list of supplementary files associated with this preprint. Click to download.

- Table5.jpg 